\title{
MARTIN KNOLL
}

\section{Dominanz als Postulat Höfische Jagd, Natur und Gesellschaft im »Absolutismus«}

Das Frontispiz der ab 1701 in vier Bänden erschienenen »Historico-Topographica Descriptio« Michael Wenings zeigt eine Allegorie auf das Kurfürstentum Bayern. Im Zentrum steht eine Frauengestalt, die Bayern symbolisiert. Sie ist von Figuren und Gegenständen umgeben, die das Land und seine Herrscherdynastie charakterisieren. Den Himmel bevölkern Heilige, die in Beziehung zum Land stehen - über ihnen allen thront die in Altötting verehrte Heilige Jungfrau Maria. Der Kurhut weist auf den politischen Status des Herrschers hin. Der Merkurstab symbolisiert das Florieren von Handel und Gewerbe, astronomische Instrumente stehen für die Wissenschaften, Kanonen versinnbildlichen die glorreiche Kriegsführung des Herrschers ${ }^{1}$.

Von besonderem Interesse sind die übrigen Figuren und Attribute, die immerhin rund die Hälfte des Bildprogramms besetzen: Sie alle stehen in Bezug zu natürlichen Ressourcen. Mythologische Figuren repräsentieren die zahlreichen Flüsse und Seen Bayerns, die mit reichen Populationen an Fisch, Krebsen und Perlmuscheln ausgestattet sind. Die allegorische Bavaria trägt Kornähren in ihrem Arm. Im Hintergrund weidet Vieh vor Pavillon- und Parkarchitektur, Wald und malerischer Landschaft. Bavaria sitzt auf einem Salzfaß. Ein anderes Bergbauprodukt, Eisen, liegt - bereits zu Barren verarbeitet - am Boden. Die Füße der Frau ruhen auf einem Marmorblock. Zuletzt fällt - prominent positioniert - reiche Jagdbeute ins Auge, Symbol für den Wildreichtum des Landes; eine Jagdwaffe, der Sauspieß, stellt den Bezug zur Jagdleidenschaft des Herrschers her.

Die panegyrische Vorrede der »Historico-Topographica Descriptio« nimmt all die im Bildprogramm thematisierten Umweltmerkmale nochmals auf und kennzeichnet sie als »natürliche Vortheil« des Landes². Die natürliche Ausstattung eines Territoriums wird in Dienst genommen für die Verherrlichung des Herrscherhauses und des Herrschers. Natur also als Argument im Herrscherlob? - Nicht nur das: Natur selbst wird zum Medium, ihre Nutzung und Transformation, ihre Domestikation dient der Inszenierung von Herrschaft und der Visualisierung von Machtanspruch. Zu studieren ist dies in der Architektur ebenso wie in der höfischen Zeremonial- und Festkultur der Frühen Neuzeit. Domestikation der Natur begegnet uns auch in der Jagdkultur früh-

1 Michael Wening, Historico-Topographica Descriptio. Das ist Beschreibung deß Churfürstenund Hertzogthumbs Ober- und Nidern Bayrn [...], Bd. 1, München 1701 (ND München 1974), (Frontispiz, unpag.); zum Bildprogramm des Titelkupfers vgl. Rainer SCHUSTER, Michael Wening und seine »Historico-Topographica Descriptio« Ober- und Niederbayerns. Voraussetzungen und Entstehungsgeschichte, München 1999 (Miscellanea Bavarica Monacensia, 171), S. 188-190.

2 Vorred an den wol-geneigten Leser, in: WenING, Historico-Topographica Descriptio (wie Anm. 1), unpag. 
neuzeitlicher Herrscher. Fürstliche Jagd markierte einen streng reglementierten privilegierten Zugriff auf natürliche Ressourcen ${ }^{3}$. Cristina Joanaz De Melo charakterisiert diese Reglementierung, die Zuteilung bzw. die Vorenthaltung von Jagdrechten, als ein wichtiges Instrument der sozialen Distinktion und der Demonstration von Herrschaft ${ }^{4}$.

Jagdausübung an frühneuzeitlichen Fürstenhöfen stand in engem funktionalem Zusammenhang mit der eben benannten Zeremonial- und Festkultur. Jagd bildete ein nicht unwesentliches Betätigungsfeld für die herrschaftliche Architektur (Jagdschlösser). Und schließlich wurden Schloßgarten und Jagdpark - wie überhaupt Schloßgarten und Territorium - nicht als streng von einander geschiedene Räume, sondern als ineinander übergehende Sphären wahrgenommen, inszeniert und genutzt ${ }^{5}$. Der Reiseschriftsteller Carl Ludwig von Pöllnitz bringt diesen Befund bezüglich des Münchener Hofes prägnant auf den Punkt, wenn er schreibt:

Doch ist unter allen churfürstlichen Lust-Schlössern Nymphenburg dasjenige, wo der Hof sich am meisten aufhält, nachdemmahlen der daranstoßende Thiergarten, so acht Meilen Umfang und verschiedene gerade ausgehauene Wege hat, dasselbe zu gleicher Zeit zu einem Lust- und jagdSchloß machet, und divertiret sich der Churfürst mit der par force Jagd darinnen, das kleine Gebüsch aber, so an den Lust-Garten stoßet, samt den Feldern um das Schloß herum, ist voller Fasanen, Feldhühner und allerhand Arten wilden Geflügels ${ }^{6}$.

3 Im Alten Reich hatte sich im Verlauf des Mittelalters ein Rechtsstatus durchgesetzt, in dem die Territorialfürsten - gestützt auf ein umstrittenes Regalitätspostulat - die jagdliche Oberhoheit für sich beanspruchten. Das oft lokal oder nach Tierarten beschränkte Jagdrecht des Adels und der Prälaten wollte man als davon abgeleitet verstanden wissen. Die Bevölkerungsmehrheit war meist von der Möglichkeit der legalen Jagdausübung ausgeschlossen. Bereits dieser Hierarchie eignete erhebliche herrschaftsdidaktische Symbolik. Vgl. Hans Wilhelm ECKARDT, Herrschaftliche Jagd, bäuerliche Not und bürgerliche Kritik. Zur Geschichte der fürstlichen und adligen Jagdprivilegien vornehmlich im südwestdeutschen Raum, Göttingen 1976 (Veröffentlichungen des Max-Planck-Instituts für Geschichte, 48), S. 37-46; zur zeitgenössischen Systematisierung vgl. Veit Ludwig VON SECKENDORFF, Teutscher Fürsten-Staat, Jena 1737 (ND Aalen 1972), S. 441f.

4 Cristina Joanaz DE Melo, Hunting. The Axis of Modern Society Organisation, in: Andrée CORvOL (Hg.), Forêt et chasse ( $\mathrm{X}^{\mathrm{e}}-\mathrm{XX} \mathrm{X}^{\mathrm{e}}$ siècle), Paris 2004, S. 57-73, hier S. 69.

5 Zur Integration von Schloßparks in ihre Umwelt durch Blickachsen und Gehölze in der barokken Gartenarchitektur vgl. Cornelia JöCHNER, Die `schöne Ordnung` und der Hof. Geometrische Gartenkunst in Dresden und anderen deutschen Residenzen, Weimar 2001, S. 67-71. Am Beispiel des fürstbischöflich Bambergischen Jagdschlosses Seehof studiert Karen Schaelow den "Zusammenklang" von Schloß, Gartenanlage und repräsentativ gestalteter (wildreicher) Umgebung als »barockes Gesamtkunstwerk « höfisch-repräsentativer Funktionalität. Karen SCHAELOW, Grünwald - Seehof, in: Zeitschrift für Bayerische Landesgeschichte 57 (1994), S. 682-693, hier S. 687-693.

6 Carl Ludwig von PÖLlnITZ, Nachrichten des Baron Carl Ludwig von Pöllnitz, Enthaltend, Was derselbe auf seinen Reisen Besonderes angemercket, Nicht weniger Die Eigenschaften derjenigen Personen, Woraus die Vornemste Höfe in Europa bestehen [...], Frankfurt a.M. 1735, Bd. 1, S. 365. 
Der hier angesprochene, 1715 fertiggestellte Jagdpark maß zunächst rund 37 Kilometer Zaunlänge und umfaßte ein Areal von 4600 Hektar; in Zeiten größter Ausdehnung erreichte er eine Fläche von 6000 Hektar?

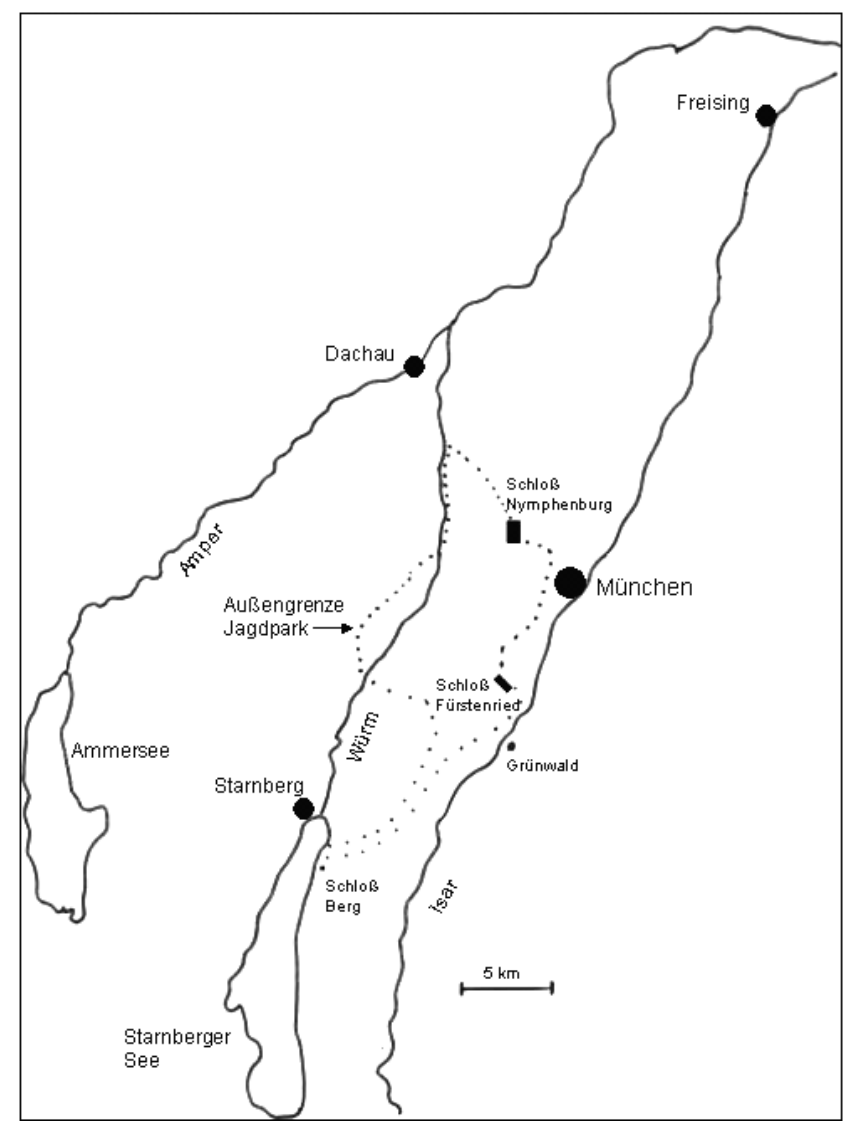

Lageskizze des kurfürstlichen Jagdparks bei München im 18. Jahrhundert in Anlehnung an Johann Baptist Tranners »Plan oder Grunt-Rüß über dem Chur Fürstlichen Hirsch jagd Parque ohn weith München [...] « (1734, Staatsarchiv München, Kartensammlung Nr. 181), und Coquilles »Plan des Environs de Starenberg \& d'une Partie du Parc de Fürstenried Servant Pour les Plaisirs de la Chasse [...] « (1716, Bayerische Staatsbibliothek München, Kartensammlung, hier nach Gerhard ScHOBER, Prunkschiffe auf dem Starnberger See. Eine Geschichte der Lustflotten bayerischer Herrscher, München 1982, S. 67).

7 Martin KNOLL, Umwelt - Herrschaft - Gesellschaft. Die landesherrliche Jagd Kurbayerns im 18. Jahrhundert, St. Katharinen 2004 (Studien zur neueren Geschichte, 4), S. 84-86. 
Im vorliegenden Sammelband wurden für die Analyse der Interaktion menschlicher Gesellschaften mit ihrer natürlichen Umwelt eine Reihe von Leitfragen entworfen, die ich wie folgt vereinfachend wiedergebe ${ }^{8}$ : erstens die Frage, inwiefern von einer Beherrschung der Umwelt durch den Menschen gesprochen werden kann, zweitens, in welcher Weise die vom Menschen beeinflußte Umwelt gesellschaftliche Machtstrukturen reflektiert, und schließlich, wie natürliche Faktoren innergesellschaftliche Machtausübung befördern oder behindern. Diesen Leitfragen werden im folgenden Diskussionsbeitrag drei konkrete Schwerpunkte zugeordnet: erstens die Frage nach Umweltsensibilität und Umweltwirksamkeit höfischer Jagd- und Festkultur, zweitens Jagdparks als spezialisierte Erlebnislandschaften und »monumentale Erweiterung[en] der fürstlichen Parkanlagen $^{9}$ und schließlich Ressourcen als die materiellen und sozialen Grenzen des absolutistischen Machtpostulats.

\section{UMWELTSENSIBILITÄT UND UMWELTWIRKSAMKEIT HÖFISCHER JAGD- UND FESTKULTUR}

In seiner methodischen Positionsbestimmung der historischen Landeskunde zwischen Landesgeschichte und historischer Geographie schlägt Erwin Riedenauer vor, den Begriff der Herrschaft als »veranlassende und formgebende Kraft bei der Gestaltung der Landschaft « ins Auge zu fassen ${ }^{10}$. Dieser stark von kulturgeographischen Arbeiten beeinflußte Ansatz besitzt insofern analytisches Potential, als er sowohl die direkt materielle Wirksamkeit (z.B. in Form von Bauten), als auch die rechtliche, soziale und im weitesten Sinne kulturelle Wirksamkeit herrschaftlichen Handelns auf die natürliche Umwelt (durch die Setzung und Durchsetzung wirtschaftlicher, sozialer und kultureller Normen und Handlungsoptionen) thematisiert. In eben diesem Kontext erscheint es sinnvoll, auch die höfische Jagd- und Festkultur frühneuzeitlicher Herrscher zu analysieren.

Die raumwirksame Mobilität der Hofgesellschaft und die Einbeziehung und Adaption landschaftlicher Gegebenheiten können anhand von Quellen höfischer Provenienz (Festbeschreibungen, Tagebücher, aber auch zeremonialtheoretische Literatur etc.) hinsichtlich Praxis und Programmatik untersucht werden. Die auf dieser Basis ermittelten Befunde haben bereits verschiedentlich raumsoziologisch argumentierende Deutungen erfahren. So nimmt Axel Schmitt Andreas Gestrichs Hinweis auf, daß das europäische Hofzeremoniell sehr stark durch die »auf den Herrscher ausgerichtete

8 Vgl. dazu die Einleitung der Herausgeber in diesem Band.

9 Rainer BECK, Ebersberg oder das Ende der Wildnis. Eine Landschaftsgeschichte, München 2003, S. 140.

10 Es sei dies, so Riedenauer, eine »geradezu exemplarische Anwendung des Forschungsbereichs >historische Landeskunde`, insofern sie eine im natürlichen Ambiente des Menschen sichtbar und greifbar gewordene Dimension seines geschichtlichen Lebens und Wirkens studiert«. Erwin RiEDENAUER, Gestaltung der Landschaft durch die Herrschaft. Begriffe, Modelle, Aspekte, in: Zeitschrift für Bayerische Landesgeschichte 57 (1994), S. 585-600, hier S. 588. 
zentralräumliche Perspektive« (Andreas Gestrich) bestimmt war ${ }^{11}$. In diesem $\mathrm{Zu}-$ sammenhang sei gerade nicht die Einteilung der Gesellschaft nach oben und unten, sondern der einheitliche Raum hervorgehoben worden, der Herrscher wie Beherrschte mit umfaßte »und in den hinein der Herrscher und seine Repräsentanten semiotisch wirken konnten« (Axel Schmitt). Im zeremonialen Zusammenhang wird die Kategorie Raum von Werner Paravicini definiert als

organisierte Abstände, zunächst zwischen Menschen, dann aber auch zwischen Gegenständen und Mauern, Gebäuden und Straßen, Fluß und Berg. Konstituierend für den Raum ist die Grenze, an der er endet und seine Qualität wechselt. Es gehört zur Raffinesse des perspektivischen Bauens der Neuzeit, daß diese Grenze systematisch negiert und damit eine illusorische Unendlichkeit der Herrschaft suggeriert wird. Raum ist im übrigen stets eine Folge der Vorstellungen und nicht umgekehrt12.

Im konkret jagdlichen Kontext weist Joseph Morsel auf den sozialen Sinn der Raumbezogenheit der Jagdausübung mittelalterlicher Herrscher hin ${ }^{13}$. Indem sich der Herrscher bei der Jagdausübung durch sein Territorium bewegt und so dessen Raum besetzt, macht er seine Herrschaft über die dort lebenden Menschen sichtbar. Alexander Schunka kann für das frühneuzeitliche Nordschwaben das Funktionieren dieser Strategie nachweisen. Er studierte Zeugenaussagen von Untertanen in jagd- und waldbedingten Reichskammergerichtsprozessen der Öttinger Grafen gegen ihre Nachbarn des 16. und 17. Jahrhunderts. Dabei zeigt er, daß die durch jagdliche Raumaneignung

11 Axel SCHMITT, Inszenierte Geselligkeit. Methodologische Überlegungen zum Verhältnis von ১Öffentlichkeitく und Kommunikationsstrukturen im höfischen Fest der Frühen Neuzeit, in: Wolfgang ADAM (Hg.), Geselligkeit und Gesellschaft im Barock, Wiesbaden 1997 (Wolfenbüttler Arbeiten zur Barockforschung, 28), S. 713-734, hier S. 731-732; Andreas GESTRICH, Absolutismus und Öffentlichkeit. Politische Kommunikation in Deutschland zur Beginn des 18. Jahrhunderts, Göttingen 1994 (Kritische Studien zur Geschichtswissenschaft, 103), S. 167.

12 Werner PARAVICINI, Zeremoniell und Raum, in: DerS. (Hg.), Zeremoniell und Raum. 4. Symposium der Residenzenkommission der Akademie der Wissenschaften zu Göttingen [...], Sigmaringen 1997 (Residenzenforschung, 6), S. 11-36, hier S. 14. An anderer Stelle wendet Cornelia JÖCHNER sich gegen eine bislang zu selten erfolgte Untersuchung des strukturellen Verhältnisses zwischen den formalen Eigenschaften der Architektur und der Sukzession des Zeremoniells. Sie stützt ihre Analyse zeremonieller Bewegung im Barockgarten u.a. auf den von Juliette HANSON und Bill HILLIER (The Social Logic of Space, Cambridge u.a. 1984) entwickelten raumtheoretischen Ansatz, der die Strukturierung von Raum als Grundvoraussetzung für Soziabilität sieht. Cornelia JöCHNER, Barockgarten und zeremonielle Bewegung. Die Möglichkeiten der Allée couverte. Oder: Wie arrangiert man ein incognito im Garten?, in: Jochen BERNS, Thomas RAHN (Hg.), Zeremoniell als höfische Ästhetik in Spätmittelalter und Früher Neuzeit, Tübingen 1995 (Frühe Neuzeit, 25), S. 470-483, hier v.a. S. 473. Zur soziologischen Interpretation von Raum als »Hauptmedium zeremonialer Hierarchisierung « am frühneuzeitlichen Fürstenhof vgl. auch: Rudolf SCHLÖGL, Der frühneuzeitliche Hof als Kommunikationsraum. Interaktionstheoretische Perspektiven der Forschung, in: Frank BECKER (Hg.), Geschichte und Systemtheorie. Exemplarische Fallstudien, Frankfurt a.M., New York 2004 (Campus Historische Studien, 37), S. 185-225, hier S. 196.

13 Joseph Morsel, Jagd und Raum. Überlegungen über den sozialen Sinn der Jagdpraxis am Beispiel des spätmittelalterlichen Franken, in: Werner RöSENER (Hg.), Jagd und höfische Kultur im Mittelalter, Göttingen 1997 (Veröffentlichungen des Max-Planck-Instituts für Geschichte, 35), S. 255-287. 
sozial konstruierte Herrschaft in das »soziale Wissen« dörflicher Gesellschaften diffundierte ${ }^{14}$. Ähnlich argumentiert Philippe Salvadori für den französischen Königshof. Er unterstreicht die Wichtigkeit der Mobilität bei der Jagdausübung und die Wichtigkeit der Infrastruktur, die diese Mobilität ermöglicht: Jagdbauten bleiben sichtbar, wenn die Hofgesellschaft schon einen Ort bereits verlassen hat. Sie verkörpern förmlich die Aktivitäten und Vorlieben des Herrschers, seine physische Existenz ${ }^{15}$. Häufig werden in diesem Zusammenhang zwei Hinweise aus der zeitgenössischen Literatur des 18. Jahrhunderts zitiert. Zum einen die Ausführungen Christian Wolffs im Kapitel »Von der Macht und Gewalt der Obrigkeit« seiner »Vernünftigen Gedancken von dem Gesellschaftlichen Leben der Menschen« aus dem Jahre 1721: Der Gemeine Mann hänge an den Sinnen und könne mangels Vernunft die Majestät und Macht des Königs nur über Dinge begreifen, die ins Auge fallen, sprich Zeremoniell und Prachtentfaltung ${ }^{16}$. Zum anderen - mit Blick auf die Jagd - finden wir im »Mundus christiano-bavaro-politicus« von 1711 die Passage, die der landesherrlichen Jagd die Funktion zuweist, daß sich die Herrscher in ihrer Ausübung überall im Lande zeigen und »auch allerorthen ihre macht durch bezaigten pracht und herrlichkeit zuerkennen geben « sollen ${ }^{17}$. Man möchte rückblickend rezeptionsgeschichtlich und im Hinblick auf die

14 Alexander SchunKA, Soziales Wissen und dörfliche Welt. Herrschaft, Jagd und Naturwahrnehmung in Zeugenaussagen des Reichskammergerichts aus Nordschwaben, 16.-17. Jahrhundert, Frankfurt a.M. u.a. 2000 (Münchener Studien zur neueren und neuesten Geschichte, 21), S.156-163.

15 Pilippe Salvadori, La chasse sous l’Ancien Régime, Paris 1996, S. 213.

16 Christian WolfF, Vernünftige Gedancken von dem Gesellschaftlichen Leben der Menschen und insonderheit dem gemeinen Wesen, Halle 1721 (ND Frankfurt a.M. 1971), S. 500f. Zur Verortung der Stellungnahme Wolffs im machtästhetischen Kontext des höfischen Zeremoniells und in der erkenntnistheoretischen Argumentation der frühneuzeitlichen ZeremonialWissenschaft vgl. Andreas GESTRICH, Höfisches Zeremoniell und sinnliches Volk. Die Rechtfertigung des Hofzeremoniells im 17. und 18. Jahrhundert, in: BERNS, RAHN (Hg.), Zeremoniell als höfische Ästhetik (wie Anm. 12), S. 56-73, hier v.a. S. 56-61. Im selben Band weist Wolfgang Weber auf die stark herrschaftskonforme Akzentuierung des Eudämonismuspostulats in der eklektischen Philosophie deutscher Aufklärer wie Julius Bernhard von Rohrs hin. Wolfgang WEBER, Zeremoniell und Disziplin. J. B. von Rohrs Ceremoniel-Wissenschaft (1728/29) im Kontext der frühneuzeitlichen Sozialdisziplinierung, in: BERNS, RAHN (Hg.), Zeremoniell als höfische Ästhetik (wie Anm. 12), S. 1-20. Marcel Thomann glaubt dagegen, die strittige Frage, ob Wolff und seine Schüler »Anhänger oder gar Funktionäre des absolutistischen Staatsgedankens« oder »Vorkämpfer der liberalen, freiheitlichen, ja revolutionären Lehren, die sich in den Ereignissen um 1789 politisch konkretisierten «, gewesen seien, eindeutig beantworten zu können: Christian Wolff sei der »frühe Verfechter des freiheitlich modernen Rechtsstaats «. Marcel Thomann, Christian Wolff, in: Michael STOLLeIS (Hg.), Staatsdenker in der Frühen Neuzeit, München ${ }^{3} 1995$, S. 257-283, hier S. 259.

17 Zit. nach Peter VolK, Die höfische Jagd, in: Hubert Glaser (Hg.), Kurfürst Max Emanuel, Bayern und Europa um 1700, Bd. 2, München 1976, S. 246. Zum »Mundus christiano-bavaropoliticus« als theoretischer Apologie des Hofes und der Politik Kurbayerns vgl. Alois SCHMID, Der »Mundus Christiano-Bavaro-Politicus«. Zur Theorie des Hofes der bayerischen Wittelsbacher im Zeitalter des höfischen Absolutismus, in: Klaus MALETTKE (Hg.), Hofgesellschaft und Höflinge an europäischen Fürstenhöfen der Frühen Neuzeit (15.-18. Jh.), Münster 2001 (Forschungen zur Geschichte der Neuzeit. Marburger Beiträge, 1), S. 125-137. Der anonyme Text wird dem familiären Umfeld des bayerischen Ratskanzlers Kaspar von Schmid (1622- 
von Christian Wolff und anderen vertretene Erkenntnistheorie vom sinnlichen Volk ${ }^{18}$ ergänzen: Prachtentfaltung als verschwenderischer Umgang mit natürlichen Ressourcen mußte innerhalb der Knappheitsgrenzen der solaren Agrargesellschaft besonders starke Signalwirkung entfalten. Jagdliche Machtdidaxe richtete sich also an den gesamten Untertanenverband ${ }^{19}$. Daneben freilich auch an andere Herrschaftsträger niedrigeren, gleichen oder höheren Ranges, inländischen Adel wie ausländische Herrscher.

Ein erstes Beispiel führt ins Elbetal bei Dresden im Sommer 161720. Der sächsische Kurfürst Johann Georg I. erwartet hohen Besuch: Kaiser Mathias in Begleitung seines Vetters, des gerade zum böhmischen König gewählten Ferdinand, und Erzherzog Maximilian haben sich angesagt. Es ist dies ein Besuch in politisch unruhiger Zeit bei einem Gastgeber, der viel Wert legt auf die Geltung seiner kurfürstlichen Präeminenz, der sich aber als kaisertreuer Protestant diplomatisch auf einem schmalen Grat bewegt $^{21}$. Der Dresdner Hof hat Schiffe elbaufwärts ins Böhmische Labasitz (Lovosice) geschickt, um den Kaiser und seinen Hofstaat dort abzuholen. Der Kurfürst selbst reist mit großem Gefolge das Elbetal hinauf zur Grenze bei Schandau, um die Gäste in Empfang zu nehmen. »Damit aber«, so lesen wir in der zeitgenössischen Schilderung Anton Wecks, »Ihr.[en] Kayserl.[ichen] und Königl.[ichen] Majestäten alle möglichste Ergötzlichkeit verschaffet werden möchte / wurde unweit Pirna am Grahl-Wäldchen auff der Elbe ein Wasser-Jagen gehalten / und darinnen 39 Stücke gefangen «22.

1693) zugeschrieben und dürfte damit von Vertrautheit mit der politischen und zeremoniellen Praxis des Münchener Hofes geprägt sein.

18 Vgl. GESTRICH, Höfisches Zeremoniell (wie Anm. 16).

19 Wie weit man der These Cristina Joanaz de Melos folgen will, daß Jagd einen der wichtigsten Faktoren darstellt, auf dem Herrschaft in neuzeitlichen Gesellschaften aufgebaut wurde, kann hier nicht abschließend diskutiert werden. Vgl. JoAnaZ DE MELO, Hunting (wie Anm. 4), S. 58.

20 Anton WECK, Der Chur-Fürstlichen Sächsischen weltberuffenen Residentz- und Haupt-Vestung Dresden Beschreib: und Vorstellung [...], Nürnberg 1680, S. 389; digitale Reproduktion des Exemplars der Herzog August Bibliothek Wolfenbüttel: http://diglib hab.de/drucke/gm-4f270/start.htm?image=00479 (Zugriff vom 11. Mai 2006).

21 Während des Besuches in Dresden erreicht der Kaiser die Zustimmung Johann Georgs zur Ausschreibung eines Kurfürstentages und seine Unterstützung für die Königswahl Ferdinands (II.) im Reich. Man trennt sich in »allen puncten eines sinnes und eines herzens«, Beratungsprotokoll, zit. nach Axel GOTTHARD, »Politice seint wir bäpstisch.« Kursachsen und der deutsche Protestantismus im frühen 17. Jahrhundert, in: Zeitschrift für Historische Forschung 20 (1993), S. 275-319, hier S. 297. Zum politischen Kontext des kaiserlichen Dresdenbesuchs vgl. auch Frank MüLLER, Kursachsen und der Böhmische Aufstand 1618-1622, Münster 1997 (Schriftenreihe der Vereinigung zur Erforschung der Neueren Geschichte, 23), S. 30f.

22 WECK, Der Chur-Fürstlichen Sächsischen weltberuffenen Residentz (wie Anm. 20), S. 389. Beim Wasser-Jagen handelte es sich um eine Sonderform der sog. Eingestellten Jagd. Für diese v.a. in Deutschland praktizierte Jagdform wurde Rot-, Schwarz- und Rehwild von Treibern in mehrtägiger bis mehrwöchiger Vorbereitung auf immer engerem Raum zusammengetrieben und in Kammern, die zuvor mit Jagdzeug (Netze, Tücher etc.) gezäunt worden waren, versammelt. Schließlich gelangte das Wild im »Lauf«, einer ebenfalls gezäunten Arena, zum Abschuß. Bei der Wasserjagd verlagerte sich dieser letzte Akt auf natürliche oder künstliche Gewässer. Vgl. dazu Gerhard STINGLWAGNER, Ilse HASEDER, Knaurs Großes Jagdlexikon, München ${ }^{4} 1996$, S. 178-180; verschiedene Gemälde höfischer Wasserjagden des 18. Jahrhunderts sind reproduziert bei Susan RichTER, Ein Zyklus und seine Pendants. Die 
Die kursächsische Festregie bespielt die landschaftliche Klaviatur der Elbe und des Elbtales virtuos. Sie liest die landschaftlichen Möglichkeiten, um sie möglichst perfekt in eine höfische Inszenierung einzubinden. Und daß eine festliche Jagd die Reise von Kurfürst und Kaiser stromabwärts unterbricht, noch bevor der Kaiser in Dresden feierlich empfangen wird, ist durchaus naheliegender Bestandteil dieser Regie. In der Zeichensprache der höfischen Repräsentation war ein gelungen inszeniertes Jagdspektakel eine Visitenkarte par excellence für den Machtanspruch und die Leistungsfähigkeit des gastgebenden Fürsten. Das Funktionieren des Gesamtarrangements zeremonieller Mobilität und Festlichkeit ist in der geschilderten Situation ohne die intensive instrumentalisierende Interaktion mit der natürlichen Umwelt nicht vorstellbar.

Eine Gegenprobe im frühen 18. Jahrhundert ermöglichen Zeremoniell und Festkultur des kurbayerischen Hofes, die durch historische, kunst- und architekturgeschichtliche Studien eingehend erforscht worden $\operatorname{sind}^{23}$. Christina Schulze etwa analysiert den Verlauf der dreiwöchigen Münchener Feierlichkeiten zur Hochzeit des bayerischen Kurprinzen Karl Albrecht mit der Habsburger Kaisertochter Maria Amalia 1722. Sie rekonstruiert dabei die Anordnung der Komponenten des Festablaufs, also Gottesdienste, Konzerte, Opernaufführungen, Bälle, Illuminationen, Feuerwerk, Schloß- und Gartenbesuche, Kutsch- und Gondelfahrten, und natürlich Jagden. Es wird eine Ablaufplanung sichtbar, die nicht zuletzt darauf angelegt ist, der Braut zunächst das Herrschaftszentrum der Münchener Residenz zu zeigen, um sie dann in Ausflügen mit der >Galaxie〈 von Jagd- und Lustschlössern ${ }^{24}$ bekanntzumachen, die um die Residenzstadt herum angeordnet sind. Im Rhythmus des Festverlaufs fällt zudem ein regelmäßiger Wechsel zwischen höfischer Aktivität im urbanen Raum von Stadt und Residenz (Gottesdienst, Oper, Ball) und einer solchen im landschaftlichen Umfeld der Jagd- und Lustschlösser auf (Jagd, Kutschfahrt, Gondelfahrt auf Kanälen, Gartenbesichtigung, Aufenthalt auf der Lustflotte auf dem Starnberger See) ${ }^{25}$.

Schwetzinger Jagdbilder, in: Die Lust am Jagen. Jagdsitten und Jagdfeste am kurpfälzischen Hof im 18. Jahrhundert, hg. von den Staatlichen Schlössern und Gärten Baden-Württemberg, Ubstadt-Weiher 1999, S. 77-93.

23 Samuel John KLINGENSMITH, The Utility of Splendor. Ceremony, Social Life and Architecture at the Court of Bavaria 1600-1800, Chicago 1993; Angela KLEIN, »Il y a plus d'aisance \& plus de divertissements «. Vorbilder und Eigenarten des Zeremoniells am Hof Max Emanuels von Bayern, in: Mitteilungen der Residenzen-Kommission der Akademie der Wissenschaften zu Göttingen 12/2 (2002), S. 33-44; Heidrun KuRZ, Barocke Prunk- und Lustschiffe am kurfürstlichen Hof zu München, München 1993 (Miscellanea Bavarica Monacensia, 163); Christina SCHUlze, Kunst und Politik am Hofe Max Emanuels. Die Hochzeit Karl Albrechts mit Maria Amalia (1722), in: Hans-Michael KÖRNER, Jürgen SCHLÄDER (Hg.), Münchner Theatergeschichtliches Symposium 2000, München 2000 (Studien zur Münchner Theatergeschichte, 1), S. 54-75; Eberhard STRAuB, Repraesentatio Majestatis oder churbayerische Freudenfeste. Die höfischen Feste in der Münchner Residenz vom 16. bis zum Ende des 18. Jahrhunderts, München 1969 (Miscellanea Bavarica Monacensia, 14); zur landesherrlichen Jagd: KNOLL, Umwelt (wie Anm. 7).

24 So die Charakterisierung, die Klingensmith, Utility of Splendor (wie Anm. 23), S. 94f., gewählt hat.

25 Ähnliche Abläufe sind auch für andere Anlässe bzw. im höfischen Alltagsleben dokumentiert, vgl. KNOLL, Umwelt (wie Anm. 7), S. 57f. 
Eine Sequenz der Feierlichkeiten am 26. Oktober verdient im Hinblick auf die zeremonielle Interaktion der höfischen Gesellschaft mit ihrer natürlichen Umwelt genaueres Hinsehen ${ }^{26}$. Am frühen Nachmittag begibt sich die Hofgesellschaft auf die Schiffe der kurfürstlichen Prunkflotte, die im Halbkreis angeordnet auf dem Starnberger See bei Schloß Berg vor Anker liegen. Die Anwesenden verteilen sich - der Hierarchie im Hofstaat folgend - auf das kurfürstliche Leibschiff, der »Bucentauro«, oder die anderen Schiffe und beobachten von dort, wie ein Hirsch ins Wasser getrieben wird und schwimmend, verfolgt von Hunden, um sein Leben kämpft. Dieser Kampf markiert das sorgsam inszenierte Finale einer Parforcejagd im bereits genannten Park, an der ein Teil der Festgesellschaft teilgenommen hatte, um dann ebenfalls an Bord zu gehen. Nach dem Tod des Tieres wird auf den Schiffen getafelt. Der Kurfürst tauft die Braut symbolisch zur Bayerin. Mit Einbruch der Dunkelheit werden an den Seeufern rings umher Illuminationen entzündet und ein Feuerwerk abgebrannt. Der Ablauf erweckt den Eindruck, daß sich in der Dramaturgie auch das für die populäre Naturwahrnehmung der Zeit nicht unwichtige Konzept der vier Elemente niedergeschlagen hat. Zwar hatte die auf Empedokles zurückgehende Lehre von den vier Elementen Erde, Wasser, Luft und Feuer als Basis allen Seins im naturwissenschaftlichen Diskurs des 18. Jahrhunderts längst an Autorität verloren27, als Gegenstand allegorischer Darstellung in Kunst, Architektur und Gartenkunst blieb sie jedoch präsent ${ }^{28}$.

Der Todeskampf des Hirschen im Wasser, von einem Festteilnehmer 1722 als »plaisir infini « beschrieben ${ }^{29}$, führt uns zum Wildtier. Tatsächlich bildet ja die ritualisierte Tötung von Wildtieren den Kern der Jagd. Simon Schama spricht - bezogen auf das anglonormannische England - von einem »Blutritual«, durch das die Hierarchie von Status und Ehre innerhalb der den König umgebenden Elite im »Kriegerstaat« geordnet worden $\mathrm{sei}^{30}$. Und eine lange Traditionslinie bildlicher wie textueller Kommunikation des Herrschaftsanspruchs jagdausübender Eliten reicht zurück bis zum Motiv des heroischen Jägers in der orientalischen Herrscherikonographie ${ }^{31}$. Alexander Schunka weist auf die Rolle der gejagten Wildtiere als soziales Medium

26 Vgl. zum folgenden ibid., S. 101.

27 Vgl. Art. »Element«, in: Johann Heinrich ZEDLER, Grosses vollständiges Universal-Lexikon Aller Wissenschaften und Künste, Bd. 8, Halle, Leipzig [...], Sp. 765-771; digitale Reproduktion: www.zedler-lexikon.de (Zugriff vom 3. Juli 2006).

28 Vgl. Hervé Brunon, Monique Mosser, Daniel RabreaU, Introduction, in: Dies. (Hg.), Les éléments et les métamorphoses de la nature imaginaire et symbolique des arts dans la culture européenne du XVI ${ }^{\mathrm{e}}$ au XVIII ${ }^{\mathrm{e}}$ siècle, Bordeaux 2004 (Annales du Centre Ledoux, 4), S. 1129, hier v.a. S. 17-26; exemplarisch zur Inszenierung der Elemente in der elisabethanischen Garten- und Festkultur im England des ausgehenden 16. Jahrhunderts und der programmatisch bedingten Dominanz des Elements Wasser: Jane AVNER, La curieuse bataille livrée entre l'eau et la terre dans un jardin Elisabéthain: Elvetham, Septembre 1591, in: BRUNON, MosSER, RABREAU (Hg.), Les éléments, S. 113-122. Vielen Dank für diesen Literaturtip an Mag. Andrea Stockhammer, Wien.

29 Pierre de Bretagne, Beichtvater des Kurfürsten Max Emanuel, zit. nach KURZ, Prunkschiffe (wie Anm. 23), S. 101.

30 Simon SchAmA, Der Traum von der Wildnis. Natur als Imagination, München 1996, S. 163.

31 Wolfram MARTINI (Hg.), Die Jagd der Eliten in den Erinnerungskulturen von der Antike bis in die Frühe Neuzeit, Göttingen 2000 (Formen der Erinnerung, 3). 
hin. Sie werden dieser Funktion gerecht, »indem sie über ihre Beziehung zum Menschen als Zeichen zwischenmenschlicher Kommunikation dienen und andererseits über symbolische Konnotationen das Verhältnis zwischen Mensch und Natur definieren « $\$ 2$.

An deutschen Fürstenhöfen des 17. und 18. Jahrhunderts ist der Umgang mit der Ressource Wildtier vom Dominanzpostulat geprägt. Jagdordnungen und Mandate formulieren eine strenge Zugriffskontrolle. Die jagdrechtliche Unterscheidung zwischen »Hochwild« und »Niederwild« spiegelt ein nach Tierarten gestuftes Prestigegefälle. Die daran orientierte Hierarchie unterschiedlicher jagdlicher Zugriffsrechte verläuft entlang der gesellschaftlichen Hierarchien.

Übrigens tendierten die kulinarischen Vorlieben der Hofküche im Münchener Beispiel diesem Gefälle durchaus zuwider ${ }^{33}$. Der adulte männliche Rothirsch etwa oder kapitale Keiler - beide besonders prestigereich als Trophäe - wurden weit weniger häufig an die Hofküche geliefert als Hirschkälber, Frischlinge oder Rehwild. Hasen, Kaninchen und Federwild gelangten fast ausschließlich auf die kurfürstliche Tafel.

Die Hege folgte der Ratio einer nur sektoralen Nachhaltigkeit, d.h. der Wildbestand sollte stets auf einem Niveau erhalten werden, wie es für die Ausübung der höfischen Jagd gebraucht wurde. Zwischen dem 10. und dem 24. November 1735 begab sich der bayerische Kurfürst Karl Albrecht (1726-1745) mit seinem Hof auf einen Jagdausflug in den Geisenfelder Forst. Bei diesem Anlaß wurden 1105 Wildschweine erlegt ${ }^{34}$. Im Jahre 1763 beging Herzog Karl Eugen von Württemberg (1744-1793) seinen Geburtstag mit zweiwöchigen Feierlichkeiten. Einen der Höhepunkte bildete ein Wasserjagen, bei dem 5000 Stück Wild zum Abschuß kamen ${ }^{35}$. Für Regionen, in denen derlei Jagdspektakel vorbereitet wurden, bedeutete dies notwendigerweise lange Zeiträume der Überhege. Diese Logik mußte in Agrargesellschaften, in denen unterschiedliche Landnutzungsoptionen in so vielen landschaftlichen Sektoren überlappten, zwangsläufig zu Konflikten führen. Lebende Tiere wurden mit großem Aufwand in Jagdparks transportiert, in Kurbayern wie anderswo ${ }^{36}$. Lebende Tiere - kapitale Hirsche zumal dienten als prestigereiche Geschenke von Fürst zu Fürst.

32 SchunKA, Soziales Wissen (wie Anm. 14), S. 156-163.

33 Dies ergab eine stichprobenartige Auswertung der Rechnungsbücher des »Wildbretgewölbes« der Münchener Residenz für den Jahrgang 1750 (Bayerisches Hauptstaatsarchiv München, Geheimes Hausarchiv, Hofjagdintendanz 142). Das Wildbretgewölbe war Umschlagplatz für einen Teil des Wildbrets aus landesherrlicher Jagd, das dort verarbeitet und entweder an die Hofküche, als »Verehrung « (Geschenk) an Adelige, Klöster, auswärtige Fürsten und Hofangehörige oder im Verkauf abgegeben wurde. Im Jahre 1750 wurden dort 1941 Stück Wild und 33 Fasaneneier umgeschlagen, darunter 120 Stück Rotwild, 160 Stück Rehwild, 96 Stück Schwarzwild, 312 Hasen, 288 Kaninchen und 962 Stück Federwild. Eine differenzierte Aufstellung bietet KNOLL, Umwelt (wie Anm. 7), S. 161-163.

34 Franz VON KoBell, Wildanger. Skizzen aus dem Gebiete der Jagd und ihrer Geschichte, mit besonderer Rücksicht auf Bayern, Stuttgart 1859, S. 128.

35 ECKARDT, Herrschaftliche Jagd (wie Anm. 3), S. 55.

36 KNOLL, Umwelt (wie Anm. 7), S. 93-97. Im England der Hannoveraner Könige Georg I. und Georg II. wurde der Wildbestand des königlichen Jagdparks im Windsor Forest nach Hofjagden stets aus anderen Reservaten ergänzt. Edward P. THOMPSON, Whigs and Hunters. The Origin of the Black Act, London 1990 ('1975), S. 234. 

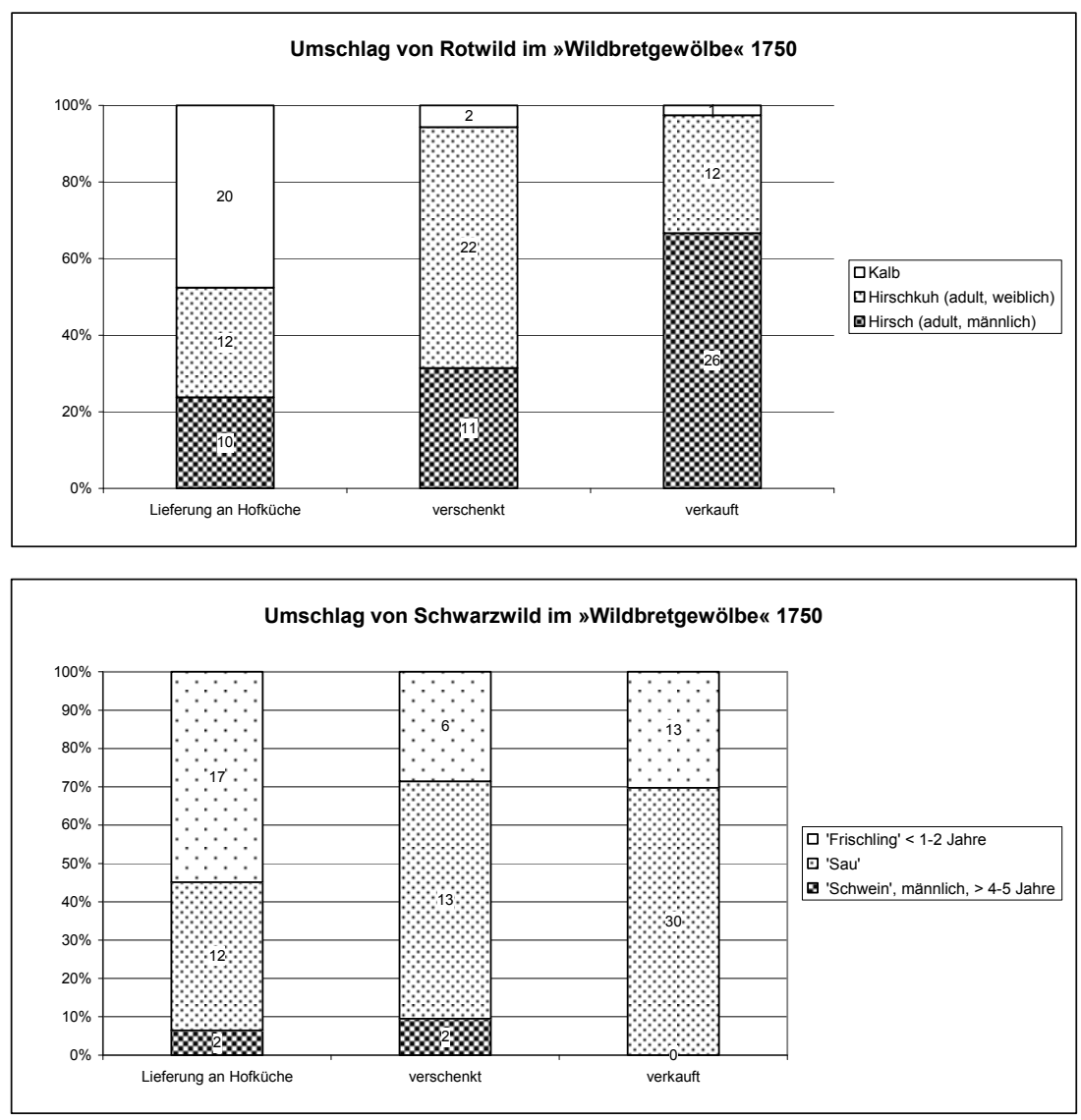

Umschlag von Hasen und Kaninchen im »Wildbretgewölbe« 1750

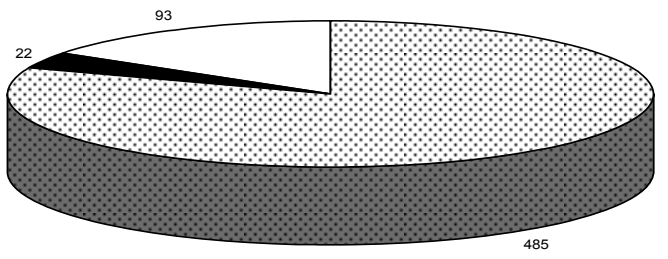




\section{JAGDPARKS: ERLEBNISLANDSCHAFTEN UND FÜRSTLICHE PARKANLAGEN IN IHRER ERWEITERUNG}

Nochmals bedarf es eines Blickes zurück auf den Schauplatz der Fest- und Jagdinszenierung des Münchener Hofes vom 26. Oktober 1722, oder konkreter: auf die Infrastruktur, ohne die diese Inszenierung nicht möglich gewesen wäre, der große kurfürstliche Jagdpark.

Rainer Beck erhält beim Studium des zeitgenössischen Kartenmaterials, das die zahlreichen axialen Wegsysteme und die mit einem Netz von Sternschneisen überzogenen Wälder dokumentiert, den Eindruck »monumentale[r] Erweiterung[en] der fürstlichen Parkanlagen ${ }^{37}$. In der Tat: Schon der räumliche Befund eines geschlossenen Jagdparks, der in Zeiten größter Ausdehnung fast 6000 Hektar Fläche umfaßte, direkt an den Nymphenburger Schloßpark angrenzte und auch zahlreiche Siedlungen einschloß, bestätigt diesen Eindruck. Rund 37 Kilometer Zaun steckten bei seiner Fertigstellung 1715 den Park als geschlossene jagdliche Erlebnislandschaft ab, die ein Jahr später durch einen gezäunten Korridor auch noch an den Starnberger See angebunden wurde, um die schon beschriebenen Jagdfinale zu ermöglichen ${ }^{38}$.

Derlei Infrastruktur läßt sich auf zwei Ebenen erklären: Die vordergründig funktionale Ebene hat mit den Ansprüchen der Jagd an die Reviergestaltung zu tun: Flora und Fauna wurden für die jagdliche Nutzung adaptiert ${ }^{39}$. Höfische Jagdtechniken, v.a. die Parforcejagd, richteten spezielle Anforderungen an die Landschaftsgestaltung: zahlreiche Wegachsen ermöglichen das problemlose Passieren auch mit Kutschen. Die Sternschneisensysteme verbessern die Einsehbarkeit des Geländes. Doch der Jagdstern - optimalerweise mit einem Jagdschloß als Zentralbau - weist auch auf die zweite, symbolisch-programmatische Ebene: Wege und Blickachsen strahlen vom Herrscher aus in alle Richtungen; das herrschaftliche Sehen in alle Richtungen wird gedoppelt im Gesehenwerden von überall her ${ }^{40}$. Hatten sich hocharistokratische Jagdgesellschaften zuvor durch mehr oder weniger >natürliche`, nur temporär durch Tücher und Netze

37 BECK, Ebersberg (wie Anm. 9), S. 140.

38 Vgl. KNOLL, Umwelt (wie Anm. 7), S. 82-93.

39 Thompson, Whigs and Hunters (wie Anm. 36), S. 236, berichtet über den Jagdpark Georgs I. im Windsor Forest von der Pflanzung von Eichenhainen als Einstand für Rotwild, von der Kornsaat zur Produktion von Wildfutter und vom Besatz des Parks mit Fasanen. Zur Adaption des Waldes von Fontainebleau als Jagdlandschaft der französischen Könige und zur Interferenz mit anderen Nutzungsformen vgl. Jean BoISsIÈRE, Exploitation Forestière et Pratiques Cynégétiques à Fontainebleau à l'Époque Moderne, in: CoRvol (Hg.), Forêt et chasse (wie Anm. 4), S. 43-56.

40 Rudolf Prinz ZUR LIPPE, Die vertikale Achse absolutistischer Herrlichkeit. Versuch über das Treppenhaus von Schloß Weißenstein, in: Die Jägerey im 18. Jahrhundert, hg. von der Arbeitsstelle Achtzehntes Jahrhundert, Bergische Universität Gesamthochschule Wuppertal, Heidelberg 1991 (Beiträge zur Geschichte der Literatur und Kunst des 18. Jahrhunderts, 11), S. 213222. JÖCHNER, Barockgarten (wie Anm. 12), S. 476-478, diskutiert im Kontext der barocken Gartentheorie die Doppelfunktion der Allee als kürzester Verbindung zwischen zwei Punkten und zugleich visueller Schiene durch den Raum. Vgl. auch Albrecht Graf VON UND ZU EGLOFFSTEIN, Jagd und Architektur, in: Die Jägerey im 18. Jahrhundert, S. 187-212. 
abgeschlossene Räume bewegt, wurde nun die Landschaft bestimmter Regionen zum jagdlichen Toporama, zur Kulisse fürstlicher Anwesenheit, umgebaut.

Im direkten Vergleich zwischen dem spätmittelalterlichen Wittelsbacher Jagdschloß Grünwald im gleichnamigen Forst unweit Münchens und der barocken fürstbischöflich bambergischen Anlage in Seehof arbeitet Karen Schaelow genau diese Akzentverschiebung heraus ${ }^{41}$ : Die Grünwalder Burganlage wird aufgrund ihrer günstigen Lage in einem wildreichen Gebiet, dennoch nahe zur Residenzstadt, durch die Wittelsbacher Herzöge seit dem 14. Jahrhundert verstärkt für die Jagd genutzt und ausgebaut. Zwar entstehen im unmittelbaren Umgriff der Burg zwei Tiergärten (von insgesamt nur 169 Tagwerk Fläche) und schreibt sich die fürstliche Jagd durch den Rodungsschutz eines großen Waldgebiets in die regionale Landschaftsentwicklung ein; dennoch handelt es sich um einen vergleichsweise begrenzten Eingriff in die natürliche Umwelt. Ganz anders dagegen das barocke Jagdschloß der Bamberger Bischöfe, das sich, so der Eindruck Schaelows, die umgebende Landschaft regelrecht unterwerfe: Inmitten prächtiger Gartenarchitektur positioniert, ist der weithin sichtbare Schloßkomplex durch breite, von Alleebäumen gesäumte Straßen mit der Region und der Residenzstadt verbunden, ordnen geometrisch angelegte Sichtschneisen Park und Umgebung. Die Verbindung von Schloß und Wald zeige sich als ein »wesentliches Element fürstlicher Lebensart und damit auch herrschaftlicher Landschaftsgestaltung « ${ }^{42}$. Der Wald trage durch die Jagd zur Pflege fürstlicher Repräsentation bei.

Philippe Salvadori analysiert den Wald von Compiègne, der seit François I. mit Sternschneisensystemen und polygonalen Wegachsen für die Jagd erschlossen worden war. 1763 hatte dieses Netzwerk eine Länge von 1600 Kilometern erreicht. Salvadori betrachtet diese elaborierte Struktur als »une démonstration géometrique de la puissance du roi sur la nature «43. Die Jagd ist Anlaß für die herrschaftliche Durchdringung des Raumes, für die Zähmung von Natur mittels geometrischer Ordnungsraster ${ }^{44}$. Die dabei evidente Affinität zu Linearität und Symmetrie schlägt eine direkte Brücke von der Landschaftsarchitektur des Jagdparks zur Gartenarchitektur des Schloßparks, dessen Herrschaftssymbolik Zeitgenossen selbstverständlich decodierten: Cay Ludwig von Hirschfelds Theorie der Gartenkunst, erschienen in fünf Bänden zwischen 1779 und 1785, stellt fest: »Durch Größe und Pracht müssen sich die Gärten der Könige und Fürsten auszeichnen« « ${ }^{5}$. Noch Friedrich Ludwig von Sckell, der die harte Linearität des Nymphenburger Schloßparks im Auftrag des Kurfürsten Karl Theodor und im Geist des englischen Landschaftsgartens überformte, gab um 1800 zu Protokoll, nur Alleen seien »imstande, Fürstengröße

41 SCHAELOW, Grünwald (wie Anm. 5).

42 Ibid., S. 692.

43 SAlVAdORI, La chasse (wie Anm. 15), S. 213f.

44 Vgl. allgemein zum Geometrismus der barocken Elitenkultur: Gernot HEISS, Die Liebe des Fürsten zur Geometrie. Adelserziehung und die Wertschätzung der höfischen Gesellschaft für Symmetrie und Regelmäßigkeit, in: Peter J. BuRGARD (Hg.), Barock. Neue Sichtweisen einer Epoche, Wien u.a. 2001, S. 101-119.

45 Zit. nach Wolfgang ADAM, Der Fürst des Wintergartens. Zur Despotismusdebatte und Gartentheorie des 18. Jahrhunderts, in: Park und Garten im 18. Jahrhundert, hg. von der Arbeitsstelle Achtzehntes Jahrhundert, Gesamthochschule Wuppertal, Heidelberg 1978 (Beiträge zur Geschichte der Literatur und Kunst des 18. Jahrhunderts, 2), S. 70-77, hier S. 70. 
durch ihren majestätischen Charakter, der ihnen ganz eigen ist, auszudrücken $\aleph^{46}$. Cornelia Jöchner charakterisiert die durch axiale Formen oder Bewuchsformationen mit der Umgebung verschalteten Schloßgärten des 16. bis 18. Jahrhunderts als komplexe Raumarrangements und »extrem künstliche Landschafts- und Architekturgebilde « ${ }^{47}$.

Norbert Elias verweist auf die kritische Sicht des Duc de Saint-Simon auf die Gartenkunst Ludwigs XIV. in Versailles. Es sei, so schreibt der Graf in seinen Memoiren, dem König ein Vergnügen, die Natur zu tyrannisieren und sie mit dem Aufgebot von Kunst und Geld zu bändigen. Man fühle sich durch den Zwang angewidert, der der Natur überall angetan werde ${ }^{48}$. Elias verweist auf den sich in Saint-Simons Äußerung manifestierenden engen Zusammenhang zwischen Herrschaftsstruktur, Parkarchitektur und Naturempfinden. Die Wendung von der zur Domestikation der Natur angewandten Kunst findet sich auch in einer anderen Quelle des 18. Jahrhunderts: Karl II. August von Zweibrücken hatte in seinem für Unsummen angelegten Schloß und Gartenkomplex Karlsberg einen aufwendigen Wintergarten erbauen lassen. Besuchern erklärte er: »Da seht Ihr, [...] wie man den Jahreszeiten vorgreifen und die Natur mit der Kunst besiegen muß«49. Von ihm selbst durchaus positiv gesehen, regte sich bei Zeitzeugen - ähnlich Saint-Simon in Versailles - der Verdacht von Despotie und Hybris ${ }^{50}$.

46 Zit. nach Martin WARNKE, Politische Landschaft. Zur Kunstgeschichte der Natur, München 1992, S. 21.

47 JÖCHNER, Schöne Ordnung (wie Anm. 5), S. 71. Dort heißt es weiter: »Höherorganisierten Zellen gleich, beginnen sie durch ihr ausgreifendes System die Landschaft zu erschließen oder ergeben Ansätze für eine neue Stadt, die nun auch regulär und geometrisch sein mußte. Hatte sich der Fürst um 1500 in eine Stadt begeben, um von einem Ort aus das Land zu regieren, so ging er um 1700 aus der befestigten und abgeschlossenen Residenzstadt heraus: die dritte Natur des Gartens, seine schöne Ordnung, griff auf das Territorium über«.

48 Zit. nach Norbert ElIAS, Die Höfische Gesellschaft. Untersuchungen zur Soziologie des Königtums und der höfischen Aristokratie [...], Neuwied 1969 (Soziologische Texte, 54), S. 338. Zur Gartenkunst des führenden Gartenarchitekten Ludwigs XIV., André Le Nôtre, vgl. Michael BRIX, Der barocke Garten. Magie und Ursprung, André Le Nôtre in Vaux-le-Vicomte, München 2004. Le Nôtres allzu gelungene Domestizierung von Natur im Garten von Vaux-le-Vicomte hatte augenscheinlich mit zum Sturz seines Auftraggebers Nicolas de Fouquet und zur Anstellung Le Nôtres durch Ludwig XIV. beigetragen. Vgl. dazu: SchamA, Der Traum von der Wildnis (wie Anm. 30), S. 364-372; Peter-Eckhard KNABE, Der Hof als Zentrum der Festkultur. Vaux-le-Vicomte, 17. August 1661, in: ADAM (Hg.), Geselligkeit und Gesellschaft im Barock (wie Anm. 11), S. 859-870.

49 Zit. nach ADAM, Fürst des Wintergartens (wie Anm. 45), S. 73. Schon in der Gartenkunst der Antike und der Renaissance wurde das Verhältnis von Natur und (menschlicher) Kunst als Kräftemessen interpretiert. Ein Besucher der Villa d'Este in Tivoli soll angesichts des Gartens seinem Gastgeber Kardinal Ippolito d'Este bewundernd versichert haben, daß die Natur hier bereitwillig zuzugestehen habe, daß sie durch die Kunst bezwungen worden sei. Claudia LAZZARO, The Italian Renaissance Garden, zit. nach Joachim WolschKE-Bulmahn, All of Germany a Garden? Changing Ideas of Wilderness in German Garden Design and Landscape Architecture, in: Christof MAUCH (Hg.), Nature in German History, New York, Oxford 2004, S. 74-92, hier S. 75f. Francis Bacon reflektierte 1623 die menschliche Fähigkeit zur Domestikation der Natur, indem er der letzteren attestierte, sie sei zwar »frei«, werde aber andererseits »von der menschlichen Kunst und Arbeit gebunden, gestaltet und gleichsam erneuert«. Zit. nach Christoph DIPPER, Deutsche Geschichte 1648-1789, Frankfurt a M. 1991 (Moderne Deutsche Geschichte, 3), S. 9f.

50 Ein weiteres gut dokumentiertes Beispiel der Inszenierung fürstlicher Macht über die Naturgewalten und die zeitgenössische Kritik daran markiert die Gestaltung der Kasseler Wilhelms- 


\section{RESSOURCEN: DIE MATERIELLEN UND GESELLSCHAFTLICHEN GRENZEN DES ABSOLUTISTISCHEN MACHTPOSTULATS}

Die Gärten Ludwigs XIV. machen auch die naturalen Grenzen des Dominanzpostulats sichtbar: Trotz aller hydraulischen Ingenieursleistungen blieb die Wasserversorgung der Fontänen im Park von Versailles prekär, mußte ein von Colbert 1672 verfaßtes Reglement genau den Ablauf fixieren, nach dem die unterschiedlichen Fontänen mit Wasser gespeist wurden, je nachdem, welche gerade im Blickfeld der königlichen Gesellschaft lag51.

Auch das hypertrophe Jagdgebaren frühneuzeitlicher Fürstenhöfe stand, was seine Abhängigkeit von natürlichen Ressourcen betrifft, auf tönernen Füßen. Hier wurde zwischen Hege und Landwirtschaft eine höchst problematische Prioritätensetzung erforderlich, die die Knappheitsgrenzen der solaren Agrargesellschaft massiv tangierte. Die Leitlinien dieser Prioritätensetzung werden am Hannoveraner Beispiel sichtbar, wo die Rechtslage in den 1780er Jahren den bäuerlichen Anspruch auf den Ersatz von Wildschäden staffelte: Je nach Entfernung vom Wald waren ein Achtel bis zwei Drittel (!) einer Ernte von vornherein als nicht ersatzfähig abzuschreiben ${ }^{52}$. Erst Wildschäden jenseits dieser Grenzen konnten geltend gemacht werden ${ }^{53}$. Eine Stellungnahme des kurbayerischen Oberstjägermeisters Franz Carl von Au aus dem Jahre 1682, in der dieser landwirtschaftliche Nutzflächen explizit als Weideflächen für das Wild einplante, folgt derselben Logik ${ }^{54}$. Entsprechend wenig kann verwundern, daß beim krisenhaften Erreichen der gesellschaftlichen Knappheitsgrenzen wie in den Hungerjahren von 1770-1773 der herrschaftliche Jagdbetrieb als Störfaktor und das Wild als Nahrungsreserve umgehend in den Focus der öffentlichen Diskussion gerieten. Nach entsprechenden Forderungen selbst der landesherrlichen Unterbehörden und unter dem Druck massiver Unruhen mußte etwa der bayerische Kurfürst Max III. Joseph verstärkten Wildabschuß und Wildbretverkauf an die notleidende Bevölkerung zu moderaten Preisen zusagen ${ }^{55}$. Der Adel wurde aufgefordert, dem Beispiel zu folgen.

höhe. Vgl. Katrin BEK, Der gelenkte Blick. Die räumliche Disposition des Friedrichsplatzes in Kassel als Seismograph gesellschaftspolitischer Veränderungen, in: Cornelia JöCHNER (Hg.), Politische Räume. Stadt und Land in der Frühneuzeit, Berlin 2003 (Hamburger Forschungen zur Kunstgeschichte, 2), S. 109-132, hier S. 118-120; WARNKE, Politische Landschaft (wie Anm. 46), S. 95f.

51 Érik ORSENNA, Portrait eines glücklichen Menschen. Der Gärtner von Versailles André Le Nôtre, 1613-1700, München 2001, S. 71f.

52 ECKARDT, Herrschaftliche Jagd (wie Anm. 3), S. 102.

53 In Pfalz-Zweibrücken waren im 18. Jahrhundert Wildschäden, die auf Flächen außerhalb der durch Wildzäune eingefriedeten Reviere entstanden, von Schadenersatzleistung gänzlich ausgeschlossen; Joachim AlLmanN, Der Wald in der frühen Neuzeit. Eine mentalitäts- und sozialgeschichtliche Untersuchung im Beispiel des Pfälzer Raumes 1500-1800, Berlin 1989 (Schriften zur Wirtschafts- und Sozialgeschichte, 36), S. 241.

54 KNOLL, Umwelt (wie Anm. 7), S. 242.

55 Helmut RANKL, Die bayerische Politik in der europäischen Hungerkrise 1770-1773, in: Zeitschrift für Bayerische Landesgeschichte 68 (2005), S. 745-779, hier S. 761f. 
Auch der Holzverbrauch jagdlicher Infrastruktur zeigt sich, bei aller Schwierigkeit, exakte Zahlen zu ermitteln, als kritische Größe. Susan Richter hat ermittelt, daß die Pfälzer Oberämter Lautern und Neustadt für den Neubau eines landesherrlichen Parforceparks bei Käfertal im Jahre 174725000 Kiefernbretter zu liefern hatten ${ }^{56}$. Daneben ist für dasselbe Projekt die Zufuhr von weiteren 12000 Brettern, 10000 Pfosten und 6000 Palisaden dokumentiert ${ }^{57}$. Ähnliche Zahlen lassen sich für den Parforcepark der bayerischen Kurfürsten bei München ermitteln, wo man wohl alleine für die Standsäulen des 37 Kilometer langen Außenzaunes im Jahre 1715 über 18000 Pfosten aus Eichenholz (oder umgerechnet rund 1212 Festmeter) benötigte ${ }^{58}$. Die begrenzte Haltbarkeit der Holzkonstruktionen erforderte dabei ständigen Ersatz ${ }^{59}$. Der herrschaftliche Jagdbetrieb verschlang Holz in mannigfaltiger Form und an vielen Orten: Parforceparks, Tiergärten, Fasanengärten etc., aber auch die Verzäunungen der landwirtschaftlichen Nutzflächen als eine der wenigen legalen Optionen bäuerlicher Wildabwehr. Treiber brannten nächtliche Feuer ab, um Wild in seiner Bewegungsfreiheit einzuschränken.

Man muß diesen Holzbedarf im Zeitkontext diskutieren. Welche Glaubwürdigkeit eignete der Argumentation der jagenden Landesherren, deren Mandate und Forstordnungen die Knappheit der Ressource Holz zur Legitimation einer immer weiter reichenden Zugriffskontrolle auf die Ressource Wald instrumentalisierten? Den ökonomisch argumentierenden Forstreformern war die offensichtliche Sorglosigkeit des Jagd- und Forstpersonals im Umgang mit Wald und Holz jedenfalls ein Greuel ${ }^{60}$.

Zwar soll hier nicht der falsche Eindruck entstehen, herrschaftliche Jagd habe sich nur innerhalb der hier diskutierten Parks abgespielt. Doch eignen sich diese hervorragend, um zu veranschaulichen, wie die herrschaftliche Jagd im späten Ancien Régime im weiteren Kontext einer zunehmenden Konflikthaftigkeit der Gestaltungshoheit bei der Nutzung natürlicher Ressourcen stand. Die Ausdehnung von Jagdparks scheint wie der allgemeine höfische Jagdaufwand im 18. Jahrhundert einen Höhepunkt zu erreichen. Die in diesem Aufsatz schon vorgestellten Gründungen bzw. Erweiterungen fränkischer, kurbayerischer und Pfälzer Anlagen warfen ähnliche Probleme auf wie die zahlreichen Neugründungen durch adelige Günstlinge König Georgs I. in England ${ }^{61}$. Stets standen eine immer größere Ausweitung herrschaftlicher Kontrolle und Inanspruchnahme der natürlichen Umwelt in Konflikt mit den angestammten Nutzungsoptionen der lokalen Bevölkerung. In diesem Zusammenhang wird auch klar,

56 Susan RichteR, Der kurfürstliche Parforce-Park in Käfertal, in: Die Lust am Jagen (wie Anm. 22), S. 43-54, hier S. 45.

57 Ibid.

58 KNOLL, Umwelt (wie Anm. 7), S. 89f.

59 Man kann davon ausgehen, daß die vom Jagdpersonal beklagten Verfallserscheinungen am Zaun des Parks im Windsor Forest eher die Regel als die Ausnahme darstellten. Vgl. THOMPSON, Whigs and Hunters (wie Anm. 36), S. 41.

$60 \mathrm{Vgl}$. etwa die kraftvoll formulierte Kritik des bayerischen Agrar- und Forstreformers Simon Rottmanner (1740-1813): Anonymus [Simon ROTTMANNER], Nothwendige Kenntnisse und Erläuterungen des Forst- und Jagdwesens in Bayern, München 1780, S. $82 \mathrm{f} ., 95$ (digitale Reproduktion online zugänglich: http://mdz1.bib-bvb.de/ db/bsb00001280/images/ [Zugriff vom 17. Juli 2006]).

61 Vgl. ThOMPSON, Whigs and Hunters (wie Anm. 36), S. 110. 
wie notwendig es ist, die Diskussion um den Stellenwert des sozialrebellischen Elements innerhalb der Wilderei ${ }^{62} \mathrm{zu}$ ökologisieren. Karl Jacoby hat mit seinem Konzept der environmental banditry eine Kategorie geschaffen, die mir auch auf die Situation im frühneuzeitlichen Europa nutzbringend anwendbar scheint. Jacoby studierte die Konflikte zwischen den Vertretern der »Conservation«-Bewegung und der ländlichen Bevölkerung in den Vereinigten Staaten des späten 19. und frühen 20. Jahrhunderts. Im Zuge der Errichtung von Naturreservaten und Nationalparks sowie einer neuen Naturschutzgesetzgebung wurden angestammte Praktiken der Naturnutzung als Mißbrauch neu definiert und kriminalisiert. Die Betroffenen reagierten auf diese Form von Umweltpolitik mit Beharren auf ihren Praktiken oder sogar deren gewaltsamen Ausweitung (environmental banditry) ${ }^{63}$.

Die hier referierten Überlegungen zu den naturalen und sozialen Grenzen eines Jagdregimes absolutistischer Prägung stecken zugleich ein Desiderat ab: Regional differenzierend wäre zu untersuchen, ob natural bedingte Faktoren der Begrenzung das Gesicht der höfischen Jagdkultur an unterschiedlichen Orten unterschiedlich prägen, oder inwieweit eine kanonische Jagdkultur auch unter regional unterschiedlichen serschwerten Bedingungen die naturalen Rahmenbedingungen oder mental-kulturelle Konzepte? Ein in seinem Fokus derart spezifizierter und empirisch orientierter regionaler Vergleich erscheint mir im übrigen vor einem generalisierenden Feststellen von korrespondierenden Phasen des historischen Wildtiermanagements und des gesellschaftlichen Naturverhältnisses stehen zu müssen, wie es mit einem tendenziell angreifbaren empirischen Fundament bereits unternommen wurde 64 .

62 Vgl. dazu in Auswahl: Ibid., S. 64-67, 190-197; ECKARDT, Herrschaftliche Jagd (wie Anm. 3), hier v.a. S. 136-141; Winfried FreITAG, Das Netzwerk der Wilderei. Wildbretschützen, ihre Helfer und Abnehmer in den Landgerichten um München im späten 17. Jahrhundert, in Andreas BlAUERT, Gerd SCHWERHOFF (Hg.), Kriminalitätsgeschichte. Beiträge zur Sozial- und Kulturgeschichte der Vormoderne, Konstanz 2000 (Konflikte und Kultur - Historische Perspektiven, 1), S. 707-757; Norbert SCHINDLER, Wilderer im Zeitalter der Französischen Revolution. Ein Kapitel alpiner Sozialgeschichte, München 2001.

$63 »$ Law and its antithesis - lawlessness - are therefore the twin axes around which the history of conservation revolves. To achieve its vision of a rational, state-managed landscape, conservation erected a comprehensive new body of rules governing the use of the environment. But to create new laws also meant to create new crimes. For many rural communities, the most notable feature of conservation was the transformation of previously acceptable practises into illegal acts: hunting or fishing redefined as poaching, foraging as trespassing, the setting of fires as arson, and the cutting of trees as thimber theft. In many cases, country people reacted to this criminalization of their customary activities with hostility. Indeed, in numerous regions affected by conservation, there arose a phenomenon, that might best be termed renvironmental banditry<, in which violations of environmental regulations were tolerated, and sometimes even supported, by members of the local rural society«. Karl JACOBY, Crimes against Nature. Squatters, Poachers, Thieves, and the Hidden History of American Conservation, Berkeley 2001, S. 2.

64 Vgl. Christoph SpEHR, Die Jagd nach Natur. Zur historischen Entwicklung des gesellschaftlichen Naturverhältnisses in USA, Deutschland, Großbritannien und Italien am Beispiel von Wildnutzung, Artenschutz und Jagd, Frankfurt a.M. 1994. 


\section{AUSBLICK}

In der 1992 von Nicholas Henshall neu befeuerten verfassungsgeschichtlichen Diskussion um den Realitätsbezug und die Brauchbarkeit des »Absolutismus«-Begriffs wurde wiederholt darauf hingewiesen, daß es sich bei der absoluten Herrschaft stets mehr um ein fürstliches Programm und staatstheoretisch fundiertes Postulat gehandelt habe, dessen Durchsetzbarkeit innerhalb der frühneuzeitlichen Gesellschaften auf einer ganz anderen Seite stand ${ }^{65}$. Für fürstliches Handeln gegenüber der Natur scheint ähnliches zu gelten: Im oben diskutierten Kontext von Jagd und Hofkultur wie in anderen Bereichen gesellschaftlicher Aktivität im Ancien Régime stößt das herrschaftliche Dominanzpostulat an enge gesellschaftliche, technische und ökologische Grenzen. Folgerichtig stand die aufgeklärte Jagdkritik des ausgehenden 18. Jahrhunderts in einem weiteren Kontext gesellschaftlicher wie ökonomischer Reformpostulate. Im 19. Jahr-hundert wendet sich vielerorts auch der aristokratische Jagdgeschmack ab von den Großspektakeln in geometrisierten Sekundärlandschaften. Der bayerische König Maximilian II. und seine Nachfolger Prinzregent Luitpold und König Ludwig III. genossen vor allem die Gebirgsjagd und posierten in volkstümlicher Jagdkleidung ${ }^{66}$. Zur gleichen Zeit mußte es anachronistisch erscheinen, wenn Kaiser Wilhelm II. ein neo-absolutistisches Jagdgepränge entwickelte - gerade wegen des Fehlens einer entsprechenden Tradition in Brandenburg-Preußen ${ }^{67}$. Wie man weiß, markierte des Kaisers jagdliche Indienstnahme der Natur nicht die einzige mißglückte Metapher seiner Herrschaft.

65 Vgl. Ronald G. Asch, Heinz DuchHARdT (Hg.), Der Absolutismus - ein Mythos? Strukturwandel monarchischer Herrschaft in West- und Mitteleuropa (ca. 1550-1700), Köln u.a. 1996 (Münsterische Historische Forschungen, 9). Die Diskussion wurde jüngst im Rahmen eines Studientages am DHI Paris engagiert fortgesetzt. Vgl. Guido BRAUN, Tagungsbericht: L'absolutisme - un concept irremplaçable? Der Absolutismus - ein unersetzliches Forschungskonzept?, in: http://hsozkult.geschichte.hu-berlin.de/tagungsberichte/id=859 (Zugriff vom 14. Juni 2006).

${ }_{66}$ Bernd E. ERGERT, Skizzen aus dem Gebiet der Jagd und ihrer Geschichte. Mit besonderer Rücksicht auf die Wittelsbacher, in: Wittelsbacher Jagd, hg. vom Deutschen Jagd- und Fischereimuseum München, München 1980, S. 9-60, hier S. 44-54.

67 Vgl. Wolfram G. THEILEMANN, Adel im grünen Rock. Adliges Jägertum, Großprivatwaldbesitz und die preußische Forstbeamtenschaft 1866-1914, Berlin 2004 (Elitenwandel in der Moderne, 5), S. 17-27. 


\section{RÉSUMÉ FRANÇAIS}

L'environnement naturel, son exploitation et sa transformation jouent, dans les panégyriques, l'architecture, le cérémonial de cour et dans les fêtes traditionnelles de l'époque moderne, un rôle significatif, lorsqu'il s'agit de mettre en scène la domination et d'évoquer la prétention au pouvoir. La manière d'ordonner et de domestiquer les paysages existants et leur morphologie, de réguler et de réduire les droits d'accès aux ressources naturelles, reflète la structure hiérarchique de la société. Dans ce contexte la pratique de la chasse par la noblesse du début de l'époque moderne mérite une attention particulière. Le contexte socioculturel de la domination »absolutiste « exige une approche plus générale qui ne réduit pas simplement la chasse à un rite de mise à mort. Cette pratique s'intégrait alors à un cycle de fêtes, c'est-à-dire à une vie quotidienne strictement réglementée par un cérémonial de cour, et avait des rapports multiples avec d'autres domaines, par exemple l'architecture des jardins. Il est possible, à partir de sources provenant des cours (descriptions de fêtes, journaux intimes) d'étudier la mobilité dans l'espace de cette société, l'intégration et l'adaptation aux réalités environnementales en tant que pratique et programme. Les résultats ainsi obtenus ont déjà fait l'objet de plusieurs interprétations se fondant sur les arguments d'une sociologie de l'espace. Le matériel administratif permet d'avoir une idée de l'usage des ressources naturelles (les animaux sauvages, le bois, etc.). L'analyse historique de l'environnement concernant la chasse pratiquée par les nobles peut tirer profit des approches développées par l'histoire des idées, de l'art et de l'architecture, qui à première vue ne traitent que de façon accessoire de la relation des sociétés humaines à l'environnement naturel. 